\title{
Key Issues in Planning and Making Sense of International Field Research
}

\author{
Susan M. Dray and David A. Siegel \\ Dray \& Associates, Inc., 2007 Kenwood Parkway, Minneapolis, Minnesota 55405 USA \\ firstname. lastnamedray.com
}

\begin{abstract}
More and more companies are doing international research as they design products and services for people around the world. These studies are particularly complex and challenging however, and there are, to date, relatively few resources to guide the people doing them. This tutorial uses scenarios and discussion to help practitioners learn how to approach, plan, manage, and interpret international field studies.
\end{abstract}

Keywords: Fieldwork, ethnography, user research, international research, user experience.

\section{Introduction}

Doing international field research is highly rewarding when it is done well, but it is fraught with challenges and complexities that can overwhelm even the most seasoned user researchers.

This tutorial focuses on key issues by posing scenarios that have been constructed to illustrate common challenges. We discuss and dissect these with participants. We also share "tips and tricks" that we have learned and found useful when doing international field research.

\section{Key Areas That Can Make or Break International Field Research}

\subsection{Making the Strategic Business Case}

Often, strategic arguments are most effective in making the case for international field research. Such arguments can focus on the strategic importance of international markets, risks of mismatches of product concepts and local conditions, or other factors of particular importance in your company.

\subsection{Preventing an Overloaded Research Agenda}

During the planning of an international user research project, user experience professionals often must struggle to keep the study from being overloaded with topics that 
either can dilute the research or can threaten to make it so hopelessly complex that it is unwieldy at best, impossible at worst.

\subsection{Team Composition and Skills}

International field research typically requires cross-cultural and often cross-functional teams. It is important to include local team members who are familiar with the culture and context. It can be equally important to include "outsiders" who are not embedded in the local culture, and people with skills in cross-cultural interpretation. Since they are be aware of the contrasts between observations and expectations, they are be able to help "message" the findings to be useful by the design teams "back home."

\subsection{Dealing with Challenges of Sampling and Recruiting}

The challenges of sampling and recruiting are complex. Criteria and screeners need to be localized and contextualized - as well as translated. You often must also deal with sampling bias that can be built into the recruiting process. The best way to deal with these issues is to treat the recruiting process as a core part of the research.

\subsection{Interpreting Findings}

Inevitably, international field researchers must make sense of "surprises" in the field. While these "surprises" are really the whole point of the research, they can present complex interpretive challenges. Sometimes, teams resort to stereotyping of the participants or jumping to simplistic conclusions about the findings. Being aware of some of the common pitfalls of interpretation and having strategies for evaluating interpretive hypotheses can help prevent these problems. 